\title{
A long, hard look at MCG-6-30-15 with XMM-Newton
}

\author{
S. Vaughan ${ }^{\text {a }}$, A. C. Fabian ${ }^{\mathrm{a}}$, K. Iwasawa ${ }^{\mathrm{a}}$ and A. K. Turner ${ }^{\mathrm{a}}$

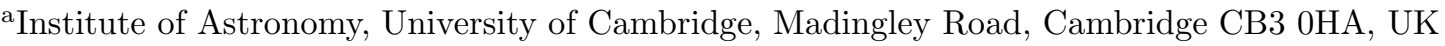

We summarise the primary results from a 320 ks observation of the bright Seyfert 1 galaxy MCG-6-30-15 with $X M M-N e w t o n$ and BeppoSAX.

\section{Introduction}

MCG-6-30-15 has received particular attention since $A S C A$ observations revealed the presence of a broad, asymmetric emission feature between $4-7 \mathrm{keV}$, identified with a highly broadened $\mathrm{Fe}$ $\mathrm{K} \alpha$ emission line ([123]). The line profile can be explained in terms of fluorescent emission from an accretion disc extending down to $\lesssim 6 r_{\mathrm{g}}$ about a black hole $\left(r_{\mathrm{g}} \equiv G M / c^{2}\right)$. This is the relativistic 'disc line' model (45). The broad $\mathrm{Fe} \mathrm{K} \alpha$ line therefore potentially offers a powerful diagnostic of the physical conditions in the immediate environment of the black hole (see [6] for a review).

However, modelling the spectrum poses considerable challenges. Along with many Seyfert galaxies, MCG-6-30-15 shows complex absorption in its X-ray spectrum. This complicates the process of identifying the correct underlying continuum and thereby measuring the superposed line emission. Indeed, the presence of relativistically broadened emission lines in the X-ray spectra of other Seyfert 1 galaxies has recently become something of a cause celebre (e.g. [7891011]).

The long $(\sim 320 \mathrm{ks})$ XMM-Newton observation of MCG-6-30-15, taken in July-August 2001 simultaneously with BeppoSAX, is of great importance since MCG-6-30-15 offers the best established example of a relativistic line profile. The results have been discussed in a series of papers (1213141511617]) and have been compared with the earlier XMM-Newton observation taken in 2000 (18]).

\footnotetext{
*Present address: X-Ray and Observational Astronomy Group, Department of Physics and Astronomy, University of Leicester, Leicester LE1 7RH, UK
}

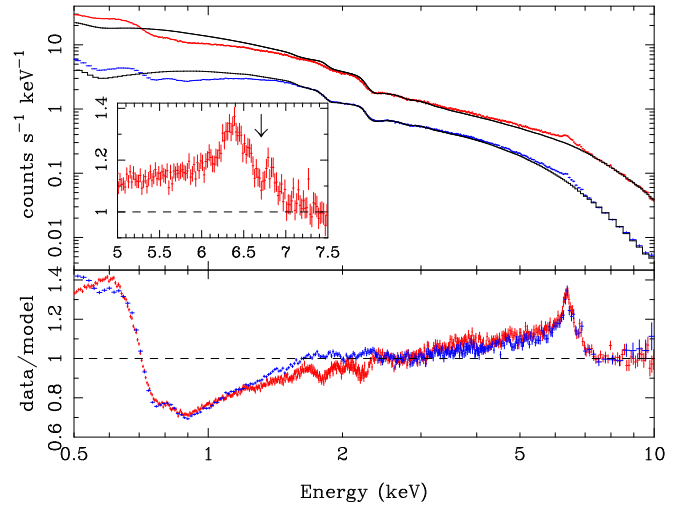

Figure 1. Top: EPIC pn and MOS spectra. Bottom: Ratio of data to a power-law model joining the $2.5-3 \mathrm{keV}$ data and $7.5-10 \mathrm{keV}$ data. See 1213 .

\section{Results}

The following are the primary observational results based on the XMM-Newton dataset.

- The two XMM-Newton observations (taken in 2000 and 2001) sampled fairly typical 'states' of the source. The former observation sampled a period of lower flux than the latter. However, the long-term $R X T E$ monitoring shows this can be attributed to short-term variability and does not imply a secular difference between the states of the source during the two observations ([15] and references therein).

- The high energy spectrum obtained from BeppoSAX shows a strong Compton-reflection signature (1217), as did the earlier $X M M$ Newton/RXTE observation (18]). There was no evidence for a low energy cut-off or roll-over in 


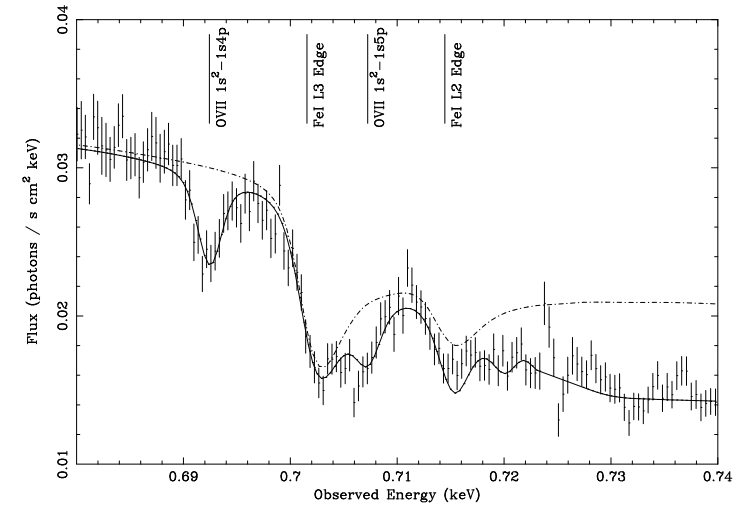

Figure 2. Close-up of the (fluxed) RGS spectrum compared to a model of O VII, O VIII and Fe I absorption (solid line) and Fe I absorption only (dot-dash line). See 16.

the continuum out to $\sim 100 \mathrm{keV}$.

- The RGS spectrum shows complex absorption including $\mathrm{O}$ VII, $\mathrm{O}$ VIII and $\mathrm{Fe}$ I and a range of other ions (19 16]; see Fig. 2). The opacity is concentrated mainly below $\sim 2 \mathrm{keV}$ but still has an effect on the $3-10 \mathrm{keV}$ spectrum (section 4.2 of [15]).

- Even after accounting for absorption the fluorescent iron line is strong and broad (121518). The bulk of the line flux is resolved with EPIC. The emission peak concentrated around $6.4 \mathrm{keV}$ is resolved with a width $F W H M \sim 4.5 \times$ $10^{4} \mathrm{~km} \mathrm{~s}^{-1}$, strongly indicating an origin within $\lesssim 100 r_{\mathrm{g}}$. There is also a significant, asymmetric extension to lower energies that indicates strong gravitational redshifts. In addition there is a weak, intrinsically narrow core to the line emission ( 1215151820 ).

- The 3-10 keV EPIC spectrum was well fitted using a model comprising emission from the surface of a relativistic accretion disc. The strong reflection explains the strength of both the iron line and the Compton reflection continuum. The best fitting model includes emission down to $\approx 1.8 r_{\mathrm{g}}$ (12 1518).

- Previous observations with $A S C A$ (23 21) and $R X T E$ ( 2223$]$ ) showed the photon index of the $2-10 \mathrm{keV}$ continuum to be correlated with

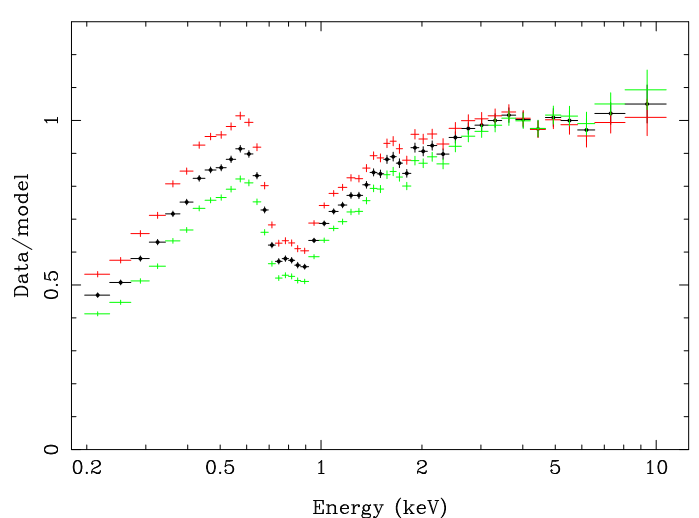

Figure 3. EPIC pn difference spectrum produced by subtracting the low flux spectrum from the high flux spectrum, shown as a ratio to a power-law (modified by Galactic absorption) fitted across the $3-10 \mathrm{keV}$ range. The difference spectrum is consistent with a power-law component (PLC) modified by warm absorption. The data/model ratio calculated using the best fitting slope is shown with circles. Above and below this lie the ratios calculated assuming the 90 per cent lower and upper limits on the slope of the $3-10 \mathrm{keV}$ power-law. See [15].

its flux. The XMM-Newton observations confirm this and demonstrate the trend is reversed below $\sim 1 \mathrm{keV}$, where the spectrum hardens with increasing flux (section 5 of [15]). The average variability amplitude is highest in the range $\sim 1-2 \mathrm{keV}$, and is lowest at energies around the iron line.

- The variable spectrum can be decomposed into two components, a variable Power-law component (PLC: Fig. 3] see [1315]) and a relatively constant Reflection Dominated Component (RDC: Fig. 4 see [1315). The spectral variability (at least on timescales $\sim 10 \mathrm{ks}$ ) can be explained almost entirely by variations in the relative strength of these two components, caused solely by changes in the normalisation of the PLC (13152124). An analysis of the flux-flux plots ( 1524$)$ and the difference spectra ( 1315$]$ ) shows the slope of the PLC remains approximately constant with flux. 


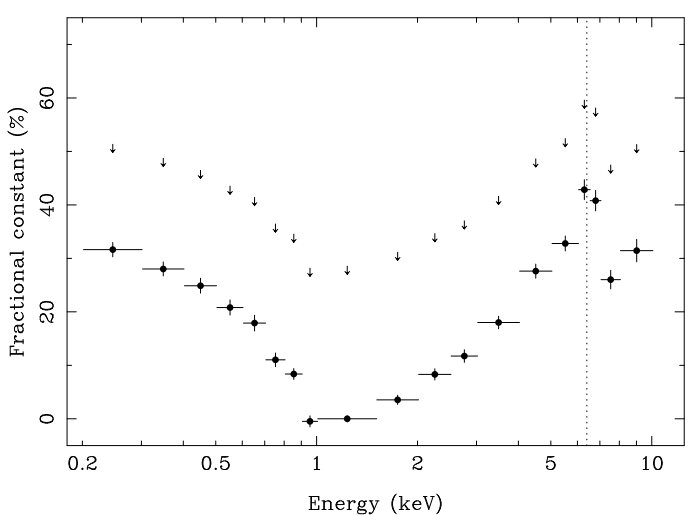

Figure 4. Fractional contribution to the spectrum from the constant component deduced from the linear flux-flux relation. The errors are $1 \sigma$ confidence limits obtained from the linear fit to the flux-flux data. This spectrum closely resembles a reflection dominated component (RDC). If the constant component contributes a non-zero fraction in the $1.0-1.5 \mathrm{keV}$ range the contributions at all energies will increase but the spectral shape will remain the same. The arrows indicate the absolute upper limits on the fraction of constant emission. See [15].

- The EPIC spectrum indicates there is resonance absorption by ionised $\mathrm{Fe}$ at $\approx 6.7 \mathrm{keV}$ (12 15] Fig. 11). This was predicted based on the presence of soft X-ray warm absorption ([19 25]) and has been observed in at least one other high quality EPIC spectrum (NGC 3783; 26]). This resonance absorption appeared to vary between the two XMM-Newton observations (section 6 of 15]).

- The variations in X-ray luminosity show many striking similarities with those seen in GBHCs such as Cygnus X-1 (14; see Fig. 6). In particular, the Power Spectral Density (PSD) function is similar to that expected by simply rescaling the high/soft state PSD of Cyg X-1. The continuum variations are energy dependent: the PSD is a function of energy and the hard variations are delayed with respect to the soft variations. Similar results have been found in other Seyfert 1s (e.g. M Hardy's article in these pro-
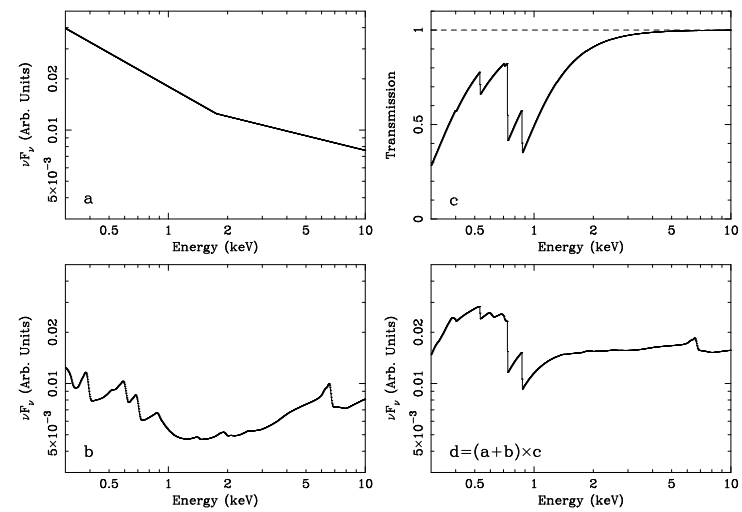

Figure 5. Schematic of the components comprising the spectral model. (a) Power-law component (PLC). (b) Reflection dominated component (RDC). (c) Dusty warm absorber (DWA). (d) Resulting observed spectrum. See [16].

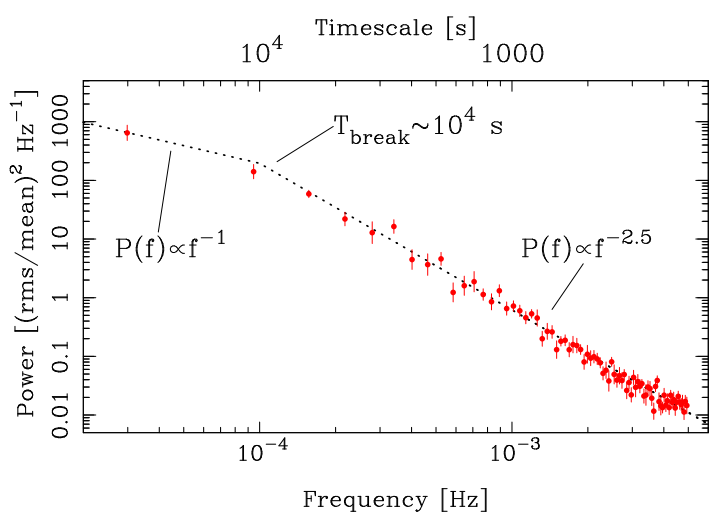

Figure 6. Power spectral density (PSD) function based on the $0.2-10 \mathrm{keV}$ EPIC pn light curve. The PSD appears steep at high frequencies and flattens towards lower frequencies. Shown is the 'unfolded' PSD. See [14.

ceedings)

\section{Conclusions}

A long observation with XMM-Newton and BeppoSAX of MCG-6-30-15 in its typical state has again confirmed the presence of the broad, skewed iron line. The best-fitting model includes 
emission from within $6 r_{\mathrm{g}}$, consistent with emission from a disk around a spinning black hole (12 18]).

The spectral variability can be explained in a phenomenological manner. The spectrum comprises the sum of two emission spectra modified by warm absorption (Fig. 5). The two emission spectra are a variable Power-Law Component (PLC) and a less variable Reflection Dominated Component (RDC). The relative constancy of the $\mathrm{RDC}$ causes variations above $\sim 2 \mathrm{keV}$ (and particularly around the iron line) to be suppressed, and also variations below $\sim 1 \mathrm{keV}$ are increasingly suppressed (12 1315]). Such a two-component emission model therefore explains the well known correlation between $2-10 \mathrm{keV}$ spectral slope and flux ([13212324]) and the relative lack of iron line variations (1523). However, the physics behind the reduced variability of the RDC is unclear. One intriguing possibility is that gravitational light bending, which may be strong if the disc extends to $\sim 2 r_{\mathrm{g}}$, is (partially) responsible (1327]).

\section{REFERENCES}

1. Tanaka Y., et al. 1995, Nature, 375, 659

2. Iwasawa K., et al. 1996, MNRAS, 282, 1038

3. Iwasawa K., Fabian A. C., Young A. J., Inoue H., Matsumoto C., 1999, MNRAS, 306, L19

4. Fabian A. C., Rees M. J., Stella L., White N. E., 1989, MNRAS, 238, 729

5. Laor A., 1991, ApJ, 376, 90

6. Reynolds C. S., Nowak M. A., 2003, Physics Reports, 377, 389

7. Lubinski P., Zdziarski A. A., 2001, MNRAS, 323, L37

8. Inoue H., Matsumoto C., 2001, AdSpR, 28, 445

9. Branduardi-Raymont G., Sako M., Kahn S. M., Brinkman A. C., Kaastra J. S., Page M. J., 2001, A\&A, 365, L140

10. Lee J. C., Ogle P. M., Canizares C. R., Marshall H. L., Schulz N. S., Morales R., Fabian A. C., Iwasawa K., 2001, ApJ, 554, L13

11. Pounds K. A., Reeves J. N., 2002, in New Visions of the X-ray Universe in the XMMNewton and Chandra Era (astro-ph/0201436)
12. Fabian A. C., et al. 2002, MNRAS, 335, L1

13. Fabian A. C., Vaughan S., 2003, MNRAS, 340, L28

14. Vaughan S., Fabian A. C,. Nandra K. 2003, MNRAS, 339, 1237

15. Vaughan S., Fabian A. C., Iwasawa K., 2003, MNRAS, in prep.

16. Turner A. K., Fabian A. C., Vaughan S., Lee J. C., 2003, MNRAS, in press (astro-ph/0303418)

17. Ballantyne D. R., Vaughan S. A., Fabian A. C., 2003, MNRAS, 342, 239

18. Wilms J., Reynolds C. S., Begelman M. C., Reeves J., Molendi S., Staubert R., Kendziorra E., 2001, MNRAS, 328, L27

19. Sako M., et al. 2003, ApJ, in press (astro-ph/0112436)

20. Lee J. C., Iwasawa K., Houck J. C., Fabian A. C., Marshall H. L., Canizares C. R., 2002, ApJ, 570, L47

21. Shih, D. C. Iwasawa, K. Fabian, A. C., 2002, MNRAS, 333, 687

22. Lee J. C., Fabian A. C., Reynolds C. S., Brandt W. N., Iwasawa K., 2000, MNRAS, 318, 857

23. Vaughan S., Edelson R. 2001, ApJ, 548, 694

24. Taylor R. D., Uttley P., McHardy I. M., 2003, MNRAS, 342, L31

25. Matt G., 1994, MNRAS, 267, L17

26. Reeves J. N., et al. , 2003, ApJ, in prep.

27. Miniutti G., Fabian A. C., Goyder R., Lasenby A. N., 2003, MNRAS, in press (astro-ph/0307163)

Based on observations obtained with XMMNewton, an ESA science mission with instruments and contributions directly funded by ESA Member States and the USA (NASA). 